\title{
Using Ark 564 and I Zw 1 as Laboratories for Modeling the Fe II Pseu0do-Continuum in QSOs
}

\author{
R. T. Eufrasio ${ }^{1,2}$, F. Bruhweiler ${ }^{1,2}$, E. Verner $^{1,2}$, B. Wills ${ }^{3}$, \\ and D. M. Crenshaw ${ }^{4}$ \\ ${ }^{1}$ The Catholic University of America \\ ${ }^{2}$ NASA Goddard Space Flight Center \\ ${ }^{3}$ University of Texas at Austin \\ ${ }^{4}$ Georgia State University
}

Keywords. accretion disks, quasars: emission lines, galaxies: Seyfert, atomic processes

In this work, we use the low-redshift, narrow-line Seyfert 1 (NLS1) galaxies Ark 564 and I Zw 1 as laboratories for modeling the Fe II emission features in the wavelength range 1200-6700 . We utilize data acquired with the Hubble Space Telescope and the 2.7-m telescope at McDonald Observatory.

We use computed grids of model Fe II emission spectra for BLR (Verner et al. 2004) and NLR (Verner et al., in preparation) clouds, and compare the spectra with the observed spectra of I Zw 1 and Akn 564. Comparisons show relatively good agreement, but also reveal Fe II lines where better atomic data are needed. The spectra of IZw 1 and Ark 564 require both BLR and NLR emission. This work confirms previous results which indicate that spectral modeling must include contributions from both BLR and NLR gas, even in the 2000-3000 where the Fe II emission from the BLR is most prominent. The largest discrepancy between predicted emission and observations is unusually strong predicted lines in the $1750-1800 \AA$ region. All of the emission lines originate at energies near $13.7 \mathrm{eV}$ and have a common lower $b^{4} G$ configuration at $6.85 \mathrm{eV}$ above ground.

The 4200-4500 $\AA$ and 4500-4700 $\AA$ emission blends gauge relative contributions of NLR and BLR gas to the Fe II spectrum (Bruhweiler \& Verner 2008). The transitions producing 4500$4700 \AA$ emission are permitted Fe II lines mostly of the $z^{4} D^{0}-b^{4} F$ and $z^{4} F^{0}-b^{4} F$ multiplets with upper and lower energy levels near 5.5 and $3.0 \mathrm{eV}$, respectively. In contrast, the 4200 $4500 \AA$ emission represents forbidden [Fe II] $a^{6} S-a^{6} D, b^{4} F-a^{6} D$, and $a^{6} G-a^{4} F$ multiplets. The lower levels for the $a^{6} D$ ground configuration have wave numbers $0-1000 \mathrm{~cm}^{-1}$ and those of $a^{4} F$ have wave numbers $2000-3000 \mathrm{~cm}^{-1}$. The [Fe II] lines weaken significantly at densities greater than $10^{6} \mathrm{~cm}^{-3}$.

So far, no devoted effort has been made to fit the spectra incorporating proper extinction, along with a good extragalactic extinction law for I Zw 1, for which we used the extinction derived by Crenshaw et al. (2002). So far we have tried only a limited range of density, ionizing radiation $(\Phi)$, and microturbulence for the BLR and NLR gas.

\section{References}

Bruhweiler, F. \& Verner, E. 2008, ApJ, 675, 83

Crenshaw, D. M., et al. 2002, ApJ, 566, 187

Verner, E., Bruhweiler, F., Verner, D., Johansson, S., Kallman, T. \& Gull, T. 2004, ApJ, 647, 676 